\title{
REVISIÓN TAXONÓMICA DE BACCHARIS SECT. HETEROTHALAMUS (ASTERACEAE: ASTEREAE) EN URUGUAY
}

\author{
VICTORIA VALTIERRA ${ }^{1}$ y JOSÉ MAURICIO BONIFACINO1
}

\begin{abstract}
Summary: Taxonomic revision of Baccharis sect. Heterothalamus (Asteracerae: Astereae) in Uruguay. A taxonomic revision of Baccharis section Heterothalamus (Less.) Giuliano (Asteraceae: Astereae) for Uruguay is presented. Baccharis sect. Heterothalamus (Less) Giuliano is composed by Baccharis aliena (Spreng.) Joch. Müll. and Baccharis psiadioides (Less.) Joch. Müll., whose geographic distributions are mostly restricted to hilly areas. This study includes a list of species, synonymy, expanded descriptions, detailed illustrations, and a key for the identification of the two species.
\end{abstract}

Key words: Asteraceae, Astereae, Baccharis, Baccharis sect. Heterothalamus, taxonomic revision, Uruguay.

\begin{abstract}
Resumen: Se presenta la revisión taxonómica de Baccharis sect. Heterothalamus (Less.) Giuliano en Uruguay. Baccharis sect Heterothalamus está representado por las especies Baccharis aliena (Spreng.) Joch. Müll. y Baccharis psiadioides (Less.) Joch. Müll., cuyas distribuciones geográficas están restringidas a las zonas de sierras. El presente estudio incluye el listado de especies, sinonimia, descripciones ampliadas, ilustraciones detalladas y una clave para la identificación de las dos especies.
\end{abstract}

Palabras clave: Asteraceae, Astereae, Baccharis, Baccharis sect. Heterothalamus, revisión taxonómica, Uruguay.

\section{INTRODUCCIÓN}

Asteraceae es la familia más numerosa a nivel mundial, la tribu Astereae es la segunda más numerosa y está representada por 222 géneros y aproximadamente 3100 especies (Funk et al., 2009; Brouillet et al., 2009).

Las Astereae se ubican principalmente en hábitats abiertos, desde zonas muy húmedas a desiertos, y desde el nivel del mar a zonas montañosas (Brouillet et al., 2009). Tradicionalmente la tribu ha sido delimitada mediante el uso de caracteres relacionados con el tipo de estilo, caracterizado por ramas estilares glabras en la cara adaxial y pubescentes en la cara abaxial, y con apéndices apicales estériles de ápice agudo. La superficie estigmática por debajo de los apéndices es conspicua y se encuentra separada en dos líneas marginales (Bremer, 1994).

1 Facultad de Agronomía, Departamento de Biología Vegetal, Laboratorio de Botánica. Casilla de Correo 1238, Montevideo, Uruguay. mvvaltierra@gmail.com; bonifacinoj@fagro.edu.uy
El género Baccharis L. es el más rico en especies dentro de la tribu Astereae, comprende entre 340 y 400 especies de distribución exclusivamente americana y se extiende desde el Sur de Estados Unidos hasta Tierra del Fuego (Giuliano, 2001; Giuliano \& Freire, 2011; Heiden 2013). Los miembros de Baccharis L. se caracterizan generalmente por la presencia de indumento en tallos y hojas y por la ocurrencia de plantas dioicas (Heiden 2013).

A pesar de la amplia distribución y de la gran diversidad morfológica de los miembros de Baccharis, la delimitación de sus taxones es muy problemática.

Si bien existe una gran diversidad de caracteres fácilmente reconocibles en las partes vegetativas (especialmente la forma de las hojas) y en los arreglos de los capítulos, la aparente homogeneidad morfológica y el reducido tamaño de los capítulos ha hecho problemática la delimitación entre especies (Müller, 2006).

La delimitación del género ha sido estudiada a lo largo de la historia por muchos autores (Cabrera, 
1974; Giuliano, 2001; Müller, 2006; Nesom, 1988) que han presentado distintas visiones del género y han ampliado o reducido la circunscripción del mismo, incluyendo o excluyendo a los géneros más afines. Recientemente se ha presentado una nueva delimitación del género en la que se amplía notablemente su circunscripción y en la que se definen nuevas Secciones.

Giuliano \& Freire (2011) presentan nuevas secciones dentro de Baccharis y proponen considerar a Heterothalamus Less. y Heterothalamulopsis Deble, A. S. Oliveira \& Marchiori como una sección dentro de Baccharis junto con la especie Baccharis boliviensis (Wedd.) Cabrera.

En Uruguay Baccharis L. es uno de los géneros más diversos de la flora nativa y el más importante de la tribu Astereae con aproximadamente 35 especies (E. Marchesi, com. pers.).

Baccharis sect. Heterothalamus (Less) Giuliano se caracteriza por presentar hojas uninervadas, receptáculos paleáceos en capítulos homógamos y corolas de las flores femeninas de tipo radio verdadero con limbos muy cortos (Giuliano et al., 2011). Está representado por cinco especies distribuidas en el centro y norte de la Argentina, sur de Brasil, sur de Perú y el norte de Chile (Deble et al., 2005; Giuliano et al., 2011; Müller 2006). En Uruguay se distribuye en el sureste, este y noreste, principalmente en zonas de sierras (Brussa \& Grela, 2007; Lombardo, 1964).

El objetivo de este trabajo es presentar la revisión taxonómica de Baccharis sect. Heterothalamus en Uruguay. Se incluye el listado de especies, sinonimia, clave de identificación de especies, descripciones ampliadas e ilustraciones detalladas.

\section{Materiales y Métodos}

Los datos presentados se obtuvieron del estudio de especímenes de herbario depositados en los herbarios: MVFA, MVJB y MVM. Adicionalmente se complementaron las observaciones en laboratorio con observaciones realizadas a campo. Se estudiaron un total de 88 caracteres morfológicos, vegetativos y reproductivos. Los datos de los caracteres morfológicos reproductivos se obtuvieron previa hidratación del material de herbario, a excepción de la forma del involucro que se determinó a campo o con material fresco.
Para cada una de las especies estudiadas se seleccionaron entre dos y tres especímenes por subdivisión político-administrativa de Uruguay (departamento) y en cada espécimen se realizaron tres mediciones para cada uno de los caracteres seleccionados. Los datos obtenidos se organizaron en tablas, a partir de las cuales se obtuvieron los rangos de los caracteres medidos. Las mediciones se realizaron con una regla de precisión $1 / 10 \mathrm{~mm}$. Las observaciones se realizaron con una lupa estereoscópica binocular Zeiss. Para la observación de tricomas, elementos del papus y márgenes de filarias, se elaboraron preparados no permanentes y se utilizó un microscopio óptico Nikon E100 con una reglilla de precisión $1 / 40 \mathrm{~mm}$.

Las ilustraciones se realizaron con cámara clara anexada a la lupa estereoscópica Nikon SMZ-10, y posteriormente fueron editadas con el programa Adobe Illustrator CS5.

Los datos registrados en las etiquetas de herbario se utilizaron para determinar la fenología, el hábitat y la distribución geográfica de las especies. Estos datos fueron complementados con datos obtenidos a campo y con material bibliográfico. Para los ejemplares tipo se transcribió la información presente en las etiquetas originales de herbario.

\section{Resultados y Discusión}

\section{Tratamiento taxonómico}

Baccharis sect. Heterothalamus (Less.) Giuliano, Ann. Missouri Bot. Gard. 98: 331 -347, 2011. Heterothalamus Less., Linnaea 5: 145, 1830; Linnaea 6: 149, 1831. Especie Tipo: Marshallia aliena Spreng., Syst. Veg. 3: 446, 1826 (= Baccharis aliena (Spreng.) Joch. Müll. Syst. Bot. Monogr. 76: 305, 2006). Heterothalamulopsis Deble, A. S. Oliveira \& Marchiori, Ci. Florest. 14: 1. 2004. Especie Tipo: Heterothalamus wagenitzii F. H. Hellwig, Haussknechtia 9: 135, 2003.

Arbustos dioicos o imperfectamente dioicos, tallos erectos, ramificados, cilíndricos, glutinosos; tallos jóvenes costados. Hojas alternas, sésiles, obovadas, elípticas a angostamente elípticas, lineares, base atenuada, ápice agudo u obtuso a agudo, glandulosas, nervio medio prominente en la cara abaxial. Capítulos agregados en inflorescencias 
terminales corimbiformes, pedunculados. Capítulos homógamos con receptáculos paleáceos, planos a convexos, alveolados. Flores femeninas con corolas de tipo radio verdadero. Aquenios fusiformes, costados. Papus 1 seriado, compuesto por cerdas barbeladas.

La historia taxonómica de Heterothalamus Less. comienza con la publicación realizada por Lessing (1830), donde presenta una breve descripción del género y basa dicha descripción en Melananthera aliena Spreng. El nombre Melananthera aliena Spreng. no corresponde a ninguna especie válidamente descripta. Un año después Lessing publica una enmienda a la publicación original del género, en la que amplía la descripción y nota que debió haber basado la descripción original en Marshallia aliena Spreng. y no en Melananthera aliena Spreng (Lessing, 1831).Adicionalmente, en ese mismo trabajo (Lessing, 1831) describe dos especies, Heterothalamus brunioides (nombre superfluo) y Heterothalmus psiadioides.

Clave para la identificación de las especies de Baccharis Sección Heterothalamus en Uruguay

1. Hojas 5,5-13 x 0,5-0,75 mm, lineares, con ápice agudo-uncinado, uninervadas, nervio hundido en la cara adaxial, margen entero a levemente crenado hacia la mitad superior, glandulosas. Aquenios glabros. .................................. B. aliena

$1^{\prime}$. Hojas 16-67 x 3,5-19 mm, obovadas o elípticas a angostamente elípticas, con ápice obtuso a agudo, margen entero en la mitad basal y aserrado hacia la mitad apical, muy glandulosas. Aquenios glandulosos. B. psiadioides

Baccharis aliena (Spreng.) Joch. Müll., Syst. Bot. Monogr. 76: 305, 2006. Marshallia aliena Spreng., Syst. Veg. 3: 446, 1826. Heterothalamus alienus (Spreng.) Kuntze, Rev. Gen. Pl. 3(3): 158, 1898. Heterothalamus brunioides Less. nom. illeg., Linnaea 6: 504, 1831. Tipo: Brasilia, [sin fecha], Sello [s.n.] (Lectotypus, aquí designado: P692145! syntypi: GH, HAL, K).

Arbustos, globosos 1-3 m de altura, tallos erectos, ramificados desde la base, cilíndricos y glutinosos; tallos jóvenes costados. Hojas alternas, espiraladas, sésiles, 5,5-13 x 0,5-0,75 mm, lineares, base atenuada, ápice agudo-uncinado, glandulosas en ambas caras, uninervadas, margen entero a levemente crenado hacia la mitad superior. Capítulos agregados en inflorescencias terminales corimbiformes, en grupos de 3 a 9, pedunculados. Capítulos heterógamos: funcionalmente masculinos, radiados, involucros 2,5-3,5 x 4-6,5 $\mathrm{mm}$, hemisféricos, filarias en 3 o 4 series; filarias externas 1,6-3 x 0,5-1 mm, angostamente elípticas, base truncada, ápice agudo, margen membranáceo, entero en la base y ciliado en el ápice, superficie glandulosa; filarias internas 2-3 x 0,5-0,75 mm, angostamente elípticas a lineares, membranáceas, base carnosa atenuada, ápice agudo, margen hialino, membranáceo entero en la base y ciliado en el ápice, tricomas glandulosos. Receptáculos epaleáceos, planos, alveolados. Flores del margen dispuestas en 1 serie, 15 a 26, femeninas, estériles; corolas tipo radios verdaderos, amarillas, tubo 0,75-1,5 mm de largo, limbo 0,75-1,2 x 0,25-0,75 $\mathrm{mm}$, elíptico a angostamente elíptico, trilobado; estilo $(0,75) 1-1,5 \mathrm{~mm}$ de largo, ramas estilares 0,25-0,5 $\mathrm{mm}$ de largo, desiguales, elípticas a lineares con ápice agudo. Flores del disco 50 a 90, hermafroditas (funcionalmente masculinas); corolas tubulosas, 5-lobadas, amarillo-verdosas, tubo 0,5-1,5 $\mathrm{mm}$ de largo, de ancho uniforme o gradualmente ensanchados hacia el ápice, lobos de ca. $0,5 \mathrm{~mm}$ de largo, angostamente elípticos de ápice agudo-atenuado; estilo 1,1-2,6 mm de largo, ramas estilares $0,5-0,55 \mathrm{~mm}$ de largo, elípticas a oblongas con ápice agudo, pubescentes en la cara abaxial, ovario rudimentario menor a $0,2 \mathrm{~mm}$ de largo; anteras 0,5-1,25 $\mathrm{mm}$ de largo, base obtusa, apéndice conectival 0,25-0,5 x 0,14-0,25 mm, ovado con ápice agudo-atenuado. Aquenios reducidos, estériles, 0,5-1,1 mm de largo, prismáticos, 3 a 5 costados, glabros. Papus 1-1,5 $\mathrm{mm}$ de largo. Capítulos homógamos: homógamos, flores fértiles con corolas de radios verdaderos, involucros 3-7 x 5,5-9 mm, globosos, acampanados, filarias en 4 a 5 series; filarias externas 1-3,5 x 0,5-1 $\mathrm{mm}$, elípticas a angostamente elípticas, carnosas, base truncada, ápice agudo, margen membranáceo, entero en la base y ciliado en el ápice, superficie abaxial con tricomas glandulosos; filarias internas 2,5-4 x 0,5-1 mm, angostamente elípticas a lineares, membranáceas, ápice agudo, base atenuada, margen hialino, membranáceo y entero, superficie glabra o glandulosa. Receptáculos paleáceos, planos a 
convexos, alveolados; paleas 2,5-4 x 0,45-0,75 $\mathrm{mm}$, angostamente elípticas a lineares, coriáceas, plegadas en forma de $\mathrm{u}$, base atenuada, ápice obtuso a agudo, margen hialino, membranáceo, ciliado hacia el ápice, superficie glabra o algo glandulosa. Flores 100 a 220, femeninas, fértiles; corolas tipo radios verdaderos, amarillas, tubo 0,75-1,5 mm de largo, limbo 0,5-0,75 x 0,3-0,5 mm, elíptico a angostamente elíptico, trilobado; estilos 1-2 $\mathrm{mm}$ de largo, ramas estilares 0,25-0,5 mm de largo, angostamente elípticas a lineares, ápice agudo, superficie estigmática en líneas marginales pubescentes. Aquenios fértiles, 1,25-2 mm de largo, fusiformes, sección triangular, 3 a 4 costados, glabros. Papus (0,75) 1-1,5 mm de largo (Fig. 1).

Distribución: En Uruguay está presente en los departamentos de Canelones, Cerro Largo, Durazno, Florida, Lavalleja, Maldonado, Río Negro, Rivera, Tacuarembó y Treinta y Tres.

Hábitat: Especie característica de ambientes serranos, presente principalmente en los departamentos de Maldonado y Lavalleja en donde constituye un elemento típico de los matorrales serranos.

Fenología: Florece de octubre a enero, fructifica de diciembre a marzo.

Lectotipificación de Marshallia aliena: Existen varios ejemplares de Marshallia aliena colectados por Sello que tienen como localidad de colecta Brasil. Estos materiales se corresponderían con material original y además, si bien se menciona como localidad de colecta solamente Brasil, éstos materiales incluirían también colectas de Montevideo (Uruguay), tal como se indica en el protólogo. De acuerdo con Stafleu y Cowan (1985) Sello depositó sus ejemplares en Berlín (B), muchos de ellos fueron destruidos durante la segunda guerra mundial, sin embargo existen duplicados en otros herbarios. De los materiales a los que se tuvo acceso, disponibles en Jstor (http://plants.jstor.org/specimen/p00692145?s=t), se seleccionó el ejemplar P692145 como lectotipo por tratarse de un ejemplar que se ajusta adecuadamente con la descripción original del nombre de la especie, con material abundante y en estado reproductivo.
Material estudiado: URUGUAY. Dpto. Canelones: Entrada a Guazubirá Viejo, 14-VII2005, Brussa \& Lafarge s.n. (MVJB, 22866); arroyo Pando, 10-X-2004, Callero s.n. (MVJB, 23045); Balneario Guazubirá y Balneario Guazubirá Nuevo, 15-XI-2007, Haretche s.n. (MVJB, 26375); límite entre Balneario Argentino y Balneario Santa Ana. 31-X-2008, Haretche s.n. (MVJB, 27066). Dpto. Cerro Largo: Sierra de Ríos, $23-$ X-1991, Bayce et. al. s.n. (MVFA, 20349). Dpto. Durazno: Cerrezuelo, 16-I-1998, González et. al. s.n. (MVFA, 27603). Dpto. Florida: Ruta 42 a $2 \mathrm{~km}$ de ruta 5, 25-III-1994, Bayce et. al. s.n. (MVFA, 24644.B); Cerro Colorado, X-1942, Aragone et. al. s.n. (MVM, PE5038). Dpto. Lavalleja: Colinas de Minas, Arechavaleta s.n. (MVM); Sierra de la Lorencita, 26-III-1996, Bayce et. al. s.n. (MVFA, 25512); Minas, Cerro del Verdún, 6-XI-1899, Berro 820 (MVFA); Minas, Cerro del Verdún, 18XII-1911, Berro 6225 (MVFA); Abra de Zabaleta, 7-V-1987, Brussa et. al. s.n. (MVJB, 20574); Cerro del Verdún, IV-1927, sin colector 2389 (MVJB); Minas, Penitente, XI-1931, Herter s.n (MVM, 22564); Penitente, 02-XI-1931, Herter 87954 (MVM); Cerro Arequita, 17-XII-1953, Legrand 4290 (MVM); Sierras de Aiguá, 20IV-1935, Legrand 599 (MVM); Serranías de Aiguá, 27-X-1940, Legrand 2283 (MVM); Minas, Arequita, X-1932, Lombardo 922 (MVJB); Cerro Arequita, 3-IV-1949, Rosengurtt 5700 (MVM). Dpto. Maldonado: Sierras de Pan de Azúcar, XI1908, Arechavaleta s.n. (MVM); Ruta 12 entre Pan de Azúcar y Minas, 12-X-1963, Arrillaga et. al. 1598 (MVFA); Abra de Perdomo, 9-XI1947, Cabrera s.n. (MVM, 13538); Sierra de las Ánimas, I-1869, Gibert 845 (MVM); Cerro de las Ánimas, 25-VI-1937, Legrand 825 (MVM); Aiguá, X-1970, Lombardo 6417 (MVJB); Cerro Pan de Azúcar, XI-1932, Lombardo 1238 (MVJB); Piriápolis, VIII-1928, Lombardo 1207 (MVJB); Sierra de las Ánimas, XII-1937, Lombardo 3141 (MVJB); Balneario Solís, 24-X-1915, Osten 7798 (MVM); Pan de Azúcar, 2-III-1920, Osten \& Schroeder 15471 (MVM); Ruta 8 hacia Aiguá, 15XII-1994, Grela et. al. s.n. (MVFA, 24293); Cerro de las Ánimas, IX-1934, Rosa Mato s.n. (MVM, 10262). Dpto. Río Negro: Cuchilla de Haedo, $22-$ XII-2006, Grela \& Haretche s.n. (MVJB, 26760); Algorta, Campo Echevarne, 24-XI-1995, Marchesi \& Vignale s.n. (MVFA, 25272). Dpto. Rivera: 

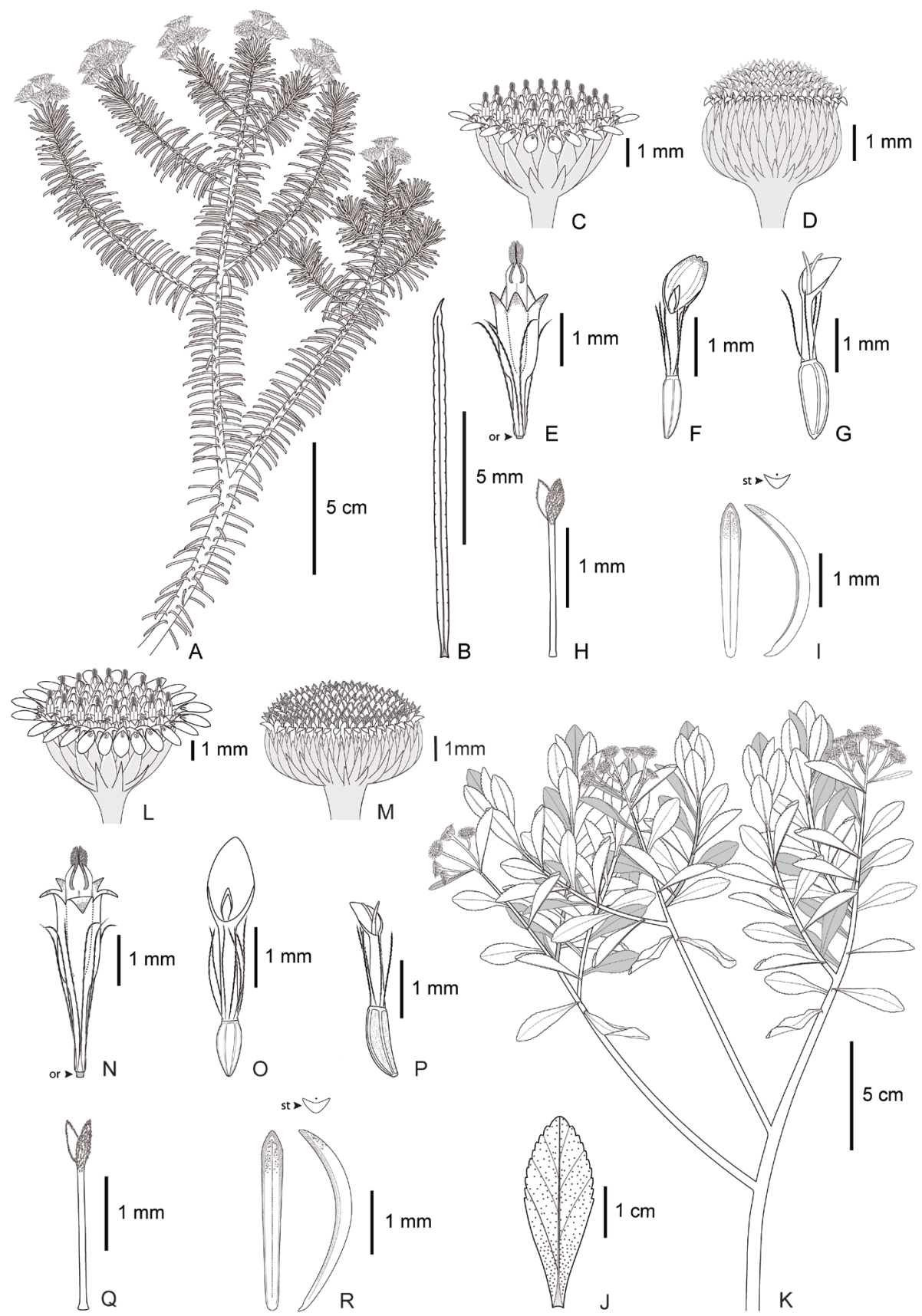

Fig. 1. A-I. Baccharis aliena. A. Hábito. B. Hoja. C. Capítulo heterógamo. D. Capítulo homógamo. E. Flor hermafrodita (funcionalmente masculina) del capítulo heterógamo. F. Flor femenina (estéril) del capítulo heterógamo. G. Flor femenina con aquenio. H. Estilo y estigma de la flor hermafrodita. I. Pálea, vista dorsal (izq.) y vista lateral (der). J-R. Baccharis psiadioides. J. Hoja. K. Hábito. L. Capítulo heterógamo. M. Capítulo homógamo. N. Flor hermafrodita (funcionalmente masculina) del capítulo heterógamo. O. Flor femenina (estéril) del capítulo heterógamo. P. Flor femenina con aquenio. Q. Estilo y estigma de la flor hermafrodita. R. Pálea, vista dorsal (izq.) y vista lateral (der.). or: ovario rudimentario; st: sección transversal. A-B: Berro 6225 (MVFA); C-E-F-H: Berro 820 (MVFA); D-G-I: González et al. 27603 (MVFA); J-K: Brussa \& Grela s.n. (MVJB 21831); L-N-O-Q: Rosengurtt B4838 (MVFA); M-P-R: Valtierra \& Bonifacino 3 (MVFA). 
arroyo del Potrero, 30-X-1997, Bonifacino et. al. s.n. (MVFA, 27430). Dpto. Tacuarembó: Cerro Agudo, 11-III-2010, Brussa \& Boggiano s.n. (MVJB); Tacuarembó, II-1956, Lombardo 5561 (MVJB). Dpto. Treinta y Tres: Quebrada de los Cuervos, 26-III-1996, Bayce et. al. s.n. (MVFA, 25539); Quebrada de los Cuervos, 16 y 18-XII1964, Brescia \& Marchesi 3775 (MVFA).

Baccharis psiadioides (Less.) Joch. Müll., Syst. Bot. Monogr. 76: 306, 2006. Heterothalamus psiadioides Less., Linnaea 6: 504, 1831. Tipo: Brasil. Rio Grande do Sul, 1833, Sello 958 [Gaudichaud]. Lectotypus, aquí designado: P604155!

Baccharis czermakii Hochr., Annuaire Conserv. Jard. Bot. Genève 3: 173, 1899. Tipus: Brasil. Rio Grande do Sul, Porto Alegre, Rua da Independencia, 10-X-1897, Czermak \& Reineck 30a (Holotipo: G, imagen!).

Arbustos ramificados, 1,2-1,7 $\mathrm{m}$ de altura, tallos erectos, cilíndricos y muy glutinosos; tallos jóvenes costados. Hojas alternas, sésiles, 16-67 x 3,5-19 $\mathrm{mm}$, se reducen gradualmente hacia el ápice, obovadas o elípticas a angostamente elípticas, base atenuada, ápice obtuso a agudo, muy glandulosas, margen entero hacia la mitad basal y aserrado hacia la mitad apical. Capítulos agregados en inflorescencias terminales corimbiformes, en grupos de 3 a 13, pedunculados. Capítulos heterógamos: funcionalmente masculinos, radiados, involucros 2-4 x 4-6,5 mm, hemisféricos, filarias en 3 o 4 series; filarias externas $2-3 \times 0,5-1 \mathrm{~mm}$, angostamente elípticas, base truncada, carnosa, ápice agudo, margen entero, membranáceo y hialino, superficie glandulosa; filarias internas 2,5-3,5 x 0,5 mm, angostamente elípticas a lineares, nervio medio marcado, castaño, base atenuada-truncada, ápice agudo-atenuado, margen entero, membranáceo y hialino, superficie glandulosa. Receptáculos epaleáceos, planos, alveolados. Flores del margen dispuestas en 1 serie, 25 a 40, femeninas, estériles; corolas tipo radios verdaderos, amarillo-verdosas, tubo 1,25-2 mm de largo, limbo 1-1,5 x 0,5-1 mm, elíptico a angostamente elíptico, trilobado; estilo 1,25-2,25 mm de largo, ramas estilares 0,2-0,5 $\mathrm{mm}$ de largo, desiguales, elípticas a angostamente elípticas con ápice agudo. Flores del disco 60 a 95, hermafroditas (funcionalmente masculinas); corolas tubulosas, 5-lobadas, amarillo-verdosas, tubo 1-2 mm de largo, de ancho uniforme o gradualmente ensanchadas hacia el ápice, lobos de ca. $0,5 \mathrm{~mm}$ de largo, angostamente elípticos de ápice agudo-atenuado; estilo 1,5-3,2 mm de largo, ramas estilares 0,5-0,6 $\mathrm{mm}$ de largo, elípticas con ápice agudo, pubescentes en la cara abaxial, ovario rudimentario menor a $0,2 \mathrm{~mm}$ de largo; anteras 0,9 $1,5 \mathrm{~mm}$ de largo, base sagitada, apéndice conectival 0,3-0,5 x 0,15-0,2 mm, angostamente elíptico a ovado con ápice atenuado. Aquenios reducidos, estériles, 0,75-1 mm de largo, prismáticos, 4 a 8 nervados. Papus 1,5-2 mm de largo. Capítulos homógamos: homógamos, flores fértiles con corolas tipo radios verdaderos, involucros 3-5 x 5-7,5 mm, globosos, acampanados, filarias en 4 o 5 series; filarias externas 1,5-3 x 0,5-1,1 mm, elípticas a angostamente elípticas, carnosas, base truncada, ápice agudo-atenuado, margen membranáceo, hialino, superficie abaxial con tricomas glandulosos; filarias internas 3-3,5 x 0,3-0,8 mm, angostamente elípticas, membranáceas, ápice agudo, base atenuada, truncada, margen membranáceo, hialino, entero hacia la base y dentado hacia el ápice, superficie glabra o glandulosa. Receptáculos paleáceos, planos a convexos, alveolados; páleas 2,5-3,3 x 0,25-0,4 mm, angostamente elípticas a lineares, planas, base truncada, ápice agudo, margen hialino, membranáceo, dentado hacia el ápice, superficie glabra. Flores 120 a 355, femeninas, fértiles; corolas tipo radios verdaderos, verde-amarillas, tubo 1-1,5 $\mathrm{mm}$ de largo, limbo $0,5-0,8 \times 0,25-0,5 \mathrm{~mm}$, elíptico a angostamente elíptico, trilobado; estilos 1,25-2 mm de largo, ramas estilares $0,25-0,5 \mathrm{~mm}$ de largo, angostamente elípticas a lineares, ápice agudo. Aquenios fértiles, 1-1,75 mm de largo, fusiformes, 4 o 5 costado, pilosos tricomas glandulosos. Papus 1-1,5 mm de largo. (Fig. 1).

Distribución: En Uruguay está presente en los departamentos de Cerro Largo, Rivera, Tacuarembó y Treinta y Tres.

Hábitat: Habita ambientes con suelos poco profundos, con alta rocosidad y buena disponibilidad de agua. Se ubica en los márgenes de bosques de quebrada y serrano, en las laderas de los cerros.

Fenología: Florece de setiembre a diciembre, fructifica de octubre a diciembre. 
Lectotipificación de Heterothalamus psiadioides: De acuerdo con el protólogo de Heterothalamus psiadioides, el ejemplar tipo se corresponde con una muestra colectada en Brasil por Sello. Como fue señalado anteriormente, los ejemplares tipo de Sello, originalmente depositados en Berlín, muy probablemente hayan sido destruídos en los incendios ocurridos en marzo de 1943. Sin embargo, hemos tenido acceso a un material, el cual a partir de la información presente en la etiqueta ha sido reconocido como material original vinculado a la descripción de esta especie. El espécimen está depositado en $\mathrm{P}$ con el número 604155. Si bien la etiqueta que figura en la muestra tiene como colector a C. Gaudichaud, de acuerdo con lo señalado por Urban (1906) el colector de la muestra sería Sello. En función de esto, y considerando que la muestra se corresponde con las caracterísicas indicadas en el protólogo, es que decidimos designar al ejemplar P604155 como lectotipo.

Material estudiado: URUGUAY. Dpto. Cerro Largo: Tupambaé, Arroyo Quebracho, 20-X-2002, Gago s.n. (MVJB 24173). Dpto. Rivera: arroyo Gajo del Lunarejo, 17-IX-1995, Bonifacino s.n. (MVFA 25045); Gruta de Piria, Cuchilla Negra, 27-X-2003, Bonifacino et. al. s.n. (MVFA 26016); Cerro Chato Dorado, 26-XII-2001, Brussa \& Escudero s.n. (MVJB 21925). Dpto. Tacuarembó: Cañada del Tala, Ruta 31, 11-XII-2010, Valtierra \& Bonifacino 3 (MVFA); Cañada del Tala y Ruta 31, 27-X-2003, Bonifacino \& Sytsma 877 (MVFA); Cerro Agudo, 11-III-2010, Brussa s.n. (MVJB); El Alero, 25-III-2010, Brussa et. al. s.n. (MVJB); Ruta 5, al sur de Paso Manuel Díaz, 11-IV-2003, Brussa \& Grela s.n. (MVJB 21831); Valle Edén, XII-1940, Chebataroff \& Lombardo 3658 (MVJB). Dpto. Treinta y Tres: Arroyo Caraja del Olimar, 10-X-1945, Rosengurtt B4838 (MVFA).

\section{Bibliografía}

BREMER, K. 1994. Asteraceae: Cladistics and classification. Timber Press, Portland, Oregon.

BROUILLET, L., T. K. LOWREY, L. URBATSCH, V. KARAMAN-CASTRO, G. SANCHO, S. WAGSTAFF \& J. C. SEMPLE. 2009. Astereae. En: Funk, V. A., A. Susanna, T. F. Stuessy \& R. J. Bayer. Systematics, Evolution, and Biogeography of Compositae, pp. 589-620. International Association for Plant Taxonomy, Vienna.
BRUSSA, C. \& I. GRELA. 2007. Flora Arbórea del Uruguay, con énfasis en las especies de Rivera $y$ Tacuarembó. COFUSA. Montevideo.

CABRERA, A. 1937. Compuestas argentinas nuevas o interesantes. Notas_Mus._La Plata, Bot. 2: 174-177.

DEBLE, L., A. OLIVEIRA \& J. N. MARCHIORI. 2005. O gênero Heterothalamus Lessing e taxones afins. Balduinia 1: 1-20.

FREIRE, S. E, A. BÁRTOLI, J. M.BONIFACINO, D.GIULIANO, G. SANCHO, N. SORIA \& R. D. TORTOSA. 2008. Asteraceae, Dicotyledoneae. En: Zuloaga, F. O., O. Morrone, M. J. Belgrano. Catálogo de las Plantas Vasculares del Cono Sur (Argentina, Sur de Brasil, Chile, Paraguay y Uruguay) Monogr. Syst. Bot. Missouri Bot. Gard.2: 1177;1343-1344.

FUNK, V. A., A. SUSANNA, T. F. STUESSY \& H. ROBINSON. 2009. Classification of Compositae. En: Funk, V. A., A. Susanna, T. F. Stuessy \& R. J. Bayer. Systematics, Evolution, and Biogeography of Compositae, pp. 171-188. International Association for Plant Taxonomy, Vienna.

GIULIANO, D. A. 2001. Clasificación infragenérica de las especies Argentinas de Baccharis (Asteraceae, Astereae). Darwiniana 39: 131-154.

GIULIANO, D. A. \& S, E. FREIRE. 2011. Nuevas secciones en Baccharis (Asteraceae: Astereae) de América del Sur. Ann. Missouri Bot. Gard. 98: 331347.

GIULIANO, D. A. \& A. PLOS 2014. Género Baccharis. En: Zuloaga, F. O., M. J. Belgrano \& A. M. Anton. Flora Argentina: flora vascular de la República Argentina. San Isidro: Instituto de Botánica Darwinion, 7: 43-123.

GIBERT,E. 1873.Enumeratio Plantarum.Heterothalamus. pp. 8. Sumptibus Socitatis. Montevideo.

MCNEILL, J., F. R. BARRIE, W. R. BUCK, V. DEMOULIN, W. GREUTER, D. L. HAWKSWORTH, P. S. HERENDEN, S. KNAPP, K. MARHOLD, J. PRADO, W. F. PRUD'HOMME VAN REINE, G. F. SMITH, J. H. WIERSEMA \& N. J. TURLAND. (eds.). 2012. International code of nomenclature for algae, fungi and plants (Melbourne Code) adopted by the eighteenth International Botanical Congress Melbourne, Australia, July 2011. Regnum Vegetabile 154. Königstein: Koeltz Scientific Books.

HEIDEN, G. 2013. Two new combinations in Baccharis, (Asteraceae:Astereae). Phytoneuron 78: 1-2.

KUNTZE, O. 1898. Revisio Generum Plantarum. Heterothalamus Less 3 (3): pp: 158. Ed. Arthur Felix. Leipzig.

LESSING, C. F. 1830. De synanthereis herbarii regii Berolinensis dissertatio secunda. Synanthereae. Heterothalamus. . Linnaea 5: pp: 145. Berlin.

LESSING, C. F. 1831. Synanthereae addendae. Heterothalamus Linnaea 6: pp: 504-505. Berlin. 
LOMBARDO, A. 1964. Flora Arbórea y Arborescente del Uruguay. $2^{\circ}$ ed. Intendencia Municipal de Montevideo.

MÜLLER, J. 2006. Systematics of Baccharis (Compositae-Astereae) in Bolivia, including overview of the genus. Syst. Bot. Monogr. 76: 1-341.

NESOM, G. L. 1988. Baccharis sect. Baccharidastrum (Compositae: Astereae), including two monoecious and one dioecious species. Phytologia 65: 169-173.

NESOM, G. L. \& H. ROBINSON. 2007. Astereae. En: Kadereit, J. W. \& C. Jeffrey (eds.), The Families and Genera of Vascular Plants. Flowering Plants. Eudicots. Asterales. Vol. 8, pp. 284-342. Springer, Berlin.

RAMAYYA, N. 1962. Studies on the tricomes of some Compositae general estructure. Bull. Bot. SURV. India: 4:177-188.

SPRENGEL, C. K. 1826. Monadelphia. Pentandria. Marshallia Systema Vegetabilium. 3: pp: 445-446Sumtibus Librariae Dieterichianae, Gottingae.
STAFLEU, F.A. \& COWAN, R.S. 1985. Taxonomic Literature: A Selective Guide to Botanical Publications with Dates, Commentaries and Types, 2nd ed., Vol: V: Sal-Ste. Regnum Vegetabile; v.112. Bohn, Scheltema \& Holkema, Utretcht.

URBAN, I. 1906. Vitae itineraque collectorum botanicorum, Notae collaboratorum biographicae, Flora Brasiliensis ratio edendi chronologica, Systema, Index Familiarum.En: Martius, C. F. P., , A. W. Eichler, \& I. Urban (eds.), Fl. Bras. Vol. 1 (1). pp: 25. Apud R. Oldenbourg in comm., Múnich.

Recibido el 19 de mayo de 2014, aceptado el 29 de julio de 2014. 